\title{
Atorvastatin Regulates MALATI/miR-200c/NRF2 Activity to Protect Against Podocyte Pyroptosis Induced by High Glucose
}

This article was published in the following Dove Press journal: Diabetes, Metabolic Syndrome and Obesity: Targets and Therapy

\author{
Yi Zuo' \\ Li Chen ${ }^{2}$ \\ Xiaoyun $\mathrm{He}^{3}$ \\ Zhen $\mathrm{Ye}^{2}$ \\ Ling $\mathrm{Li}^{\mathrm{I}}$ \\ Zhanhong Liu' \\ Suxian Zhou' \\ 'Department of Endocrinology, Affiliated \\ Hospital of Guilin Medical University, \\ Guilin, 54I00I, People's Republic of \\ China; ${ }^{2}$ Guangxi Key Laboratory of Brain \\ and Cognitive Neuroscience, Guilin, \\ Guangxi, 541004, People's Republic of \\ China; ${ }^{3}$ Department of Endocrinology, \\ Xiangya Hospital, Central South \\ University, Changsha, 410008, People's \\ Republic of China
}

Background: Diabetic nephropathy (DN) is one of the main complications of diabetes mellitus (DM), which leads to the long-term loss of kidney functions. Long noncoding RNAs (LncRNAs) can alleviate DN by interacting with microRNAs (miRNAs). In this work, we aimed to explore the effects of the MALAT1/miR-200c/NRF2 regulatory axis on the pyroptosis and oxidative stress (Oxidative stress, OS) of renal podocytes in high glucose (HG) environment and whether the lipid-lowering drug atorvastatin (AT) can relieve renal OS through this approach.

Methods: MPC-5, a mouse podocyte cell line, was induced by HG as a cell model. The protein expressions of caspase-1, GSDMD, NLRP3, NRF2, etc. were detected by Western blotting and immunofluorescence, and the mRNA level of caspase-1, GSDMD, NLRP3, NRF2, MALAT1, miR-200c was tested by qRT-PCR. The cell pyroptosis of podocytes treated with AT was verified by CCK-8 or flow cytometry. The levels of Malondialdehyde (MDA), superoxide dismutase (SOD), and glutathione (GSH) were measured by spectrophotometer, respectively.

Results: The caspase-1 was upregulated in time-dependent manner and got the peak at 48 $\mathrm{h}$ and $30 \mathrm{mmol} / \mathrm{L}$ respectively in MPC-5 cells treated with HG. Further, the expression of GSDMD, MALAT1 and miR-200c were increased, while the level of NRF2, HO-1, OSrelated indicators, were decreased simultaneously. Knockdown the MALAT1 protected MPC-5 cells from pyroptosis and OS induced by HG. However, overexpressing miR-200c in control-group cells increased pyroptosis and upregulated the OS level with HG culture medium. Further, atorvastatin protected MPC-5 cells from cell pyroptosis and downregulated the level of renal OS via attenuating the expression of MALAT1 and miR-200c.

Conclusion: Atorvastatin protects podocyte cells via MALAT1/miR-200c/NRF2 signal pathway from pyroptosis and OS induced by HG.

Keywords: atorvastatin, MALAT1, miR-200c, NRF2, podocytes, pyroptosis

\section{Introduction}

Diabetic nephropathy (DN) is a serious, chronic microvascular complication of diabetes mellitus (DM), which is the main cause of end-stage renal disease (ESRD) and diabetes mortality. ${ }^{1}$ Its specific pathogenesis remains unclear, but proteinuria is one of the clinical signs of DN and is associated with damage of the glomerular filtration barrier (GFB). Podocytes, highly differentiated cells in the outermost layer of the GFB, whose damage is related to the destruction of the GFB and the occurrence of proteinuria. ${ }^{2-4}$ Therefore, the investigation of podocytes has become a hot spot to explore the pathogenesis of DN. Pyroptosis, a newly
Correspondence: Suxian Zhou Department of Endocrinology, Affiliated Hospital of Guilin Medical University, 15 Lequn Road, Guilin, Guangxi, 54I00I, People's Republic of China Email zoe_doctor@163.com
Diabetes, Metabolic Syndrome and Obesity: Targets and Therapy 2021:14 1631-1645

$163 \mid$

DovePress $f$ in $\boldsymbol{v}$


discovered way of programmed cell death, is accompanied by the release of inflammatory factors including interleukin (IL)-1 $\beta$, IL-18, etc. ${ }^{2}$ The morphological changes of pyroptosis are similar to necrosis and apoptosis. ${ }^{5-7}$ It is a form of programmed cell death that depends on caspase-1, which induces an inflammatory response through a cascade of reactions. ${ }^{5}$ Studies of the importance of pyroptosis in cancer, cardiovascular diseases, and microbial infectious diseases are ongoing. ${ }^{8-10}$

Previous studies have confirmed that cell pyroptosis is relevant to oxidative stress (OS). Recent in vivo studies reported an association between OS and podocyte damages that resulted in albuminuria, the activation of NLRP3 inflammasomes in the glomerular cortex, and increased production of IL-1 $\beta$. The evidence indicates that podocyte pyroptosis may be a response to OS. ${ }^{11}$ Nuclear factor erythroid-2-related factor 2 (NRF2) participates in the regulation of OS and its expression is downregulated under high glucose (HG) condition. ${ }^{12} \mathrm{Hu}$ et $\mathrm{al}^{13}$ found that the mice knocked out NRF2 are more sensitive to oxidative damage and respond with an increase in pyroptosis. NRF2 regulates OS-induced pyroptosis via heme oxygenase-1 (HO-1), an antioxidant enzyme that is involved in regulating antioxidant and antiinflammatory responses in cells. ${ }^{14}$ In vitro studies ${ }^{15,16}$ have confirmed that the NRF2/HO-1 regulatory pathway is of key significance in the occurrence and development of DN. Metastasis-related lung adenocarcinoma transcript 1 (MALAT1) is a highly conserved long noncoding RNA (lncRNA) that has been found to be associated with pyroptosis in DM. Overexpression of MALAT1 significantly inhibits the pyroptosis of human umbilical vein endothelial cells induced by $\mathrm{H}_{2} \mathrm{O}_{2}$, leading to the stabilization and activation of NRF2. ${ }^{17}$ MicroRNAs (MiRNAs), important signal regulatory molecules in cells, are endogenous noncoding single-stranded RNA that have 20-24 nucleotides. Elevated expression of miR-200c can increase the level of OS in endothelial cells. ${ }^{18}$ Moreover, Li et $\mathrm{al}^{19}$ found that miR-200c can combine with MALAT1 in the structure.

In addition to lipid regulation, statins have antiinflammatory and antioxidative effects. For example, atorvastatin (AT) was reported to increase superoxide dismutase (SOD) and glutathione peroxidase (GSH-Px) expression and decrease glutathione peroxidase (GSH-Px) expression in rats with streptozotocin (STZ)-induced kidney injury. The protective effect of AT on the kidney may be related to its reduction of renal OS, but the specific mechanism is unknown. $^{20}$
However, related studies have confirmed that excessive doses of statins worsen the blood sugar control of diabetic patients. $^{21,22}$ And the risk of new-onset diabetes caused by high-dose statins is higher than that of low-dose statins. ${ }^{23}$ Thongtang et $\mathrm{al}^{24}$ found that compared with patients who continued to take low-dose simvastatin, the glucose homeostasis of diabetic patients who switched from low-dose simvastatin to high-dose atorvastatin decreased slightly, and $\mathrm{HbAlc}$ increased by $0.1 \%$. A randomized controlled trial showed that a dose of $80 \mathrm{mg} /$ day of atorvastatin significantly increased the median insulin level, indicating that the deterioration of blood glucose control is caused by increased insulin resistance. ${ }^{25}$ Henriksbo et $\mathrm{al}^{26}$ found that the combination of statins, $\mathrm{p} 38$ and mTOR promoted the production of IL-1 $\beta$, which is an NLRP3 inflammasome effector that facilitates insulin resistance in adipocytes. These indicated that statins reduce the isoprenoid required for protein isoprenylation and activate NLRP3, which in turn generates IL-1 $\beta$ to accelerate insulin resistance in adipocytes. $^{27,28}$

This study investigated whether the MALAT1/miR200c/NRF2 axis regulated OS and podocyte pyroptosis in DN induced by HG and whether the protective effect of AT shown by improvement of podocyte pyroptosis was mediated by the MALAT1/miR-200c/NRF2 axis.

\section{Methods}

\section{Cell Culture and HG Induction}

Mouse podocyte cells, MPC-5, were purchased from Shanghai Aolu Limited and were cultured in RMPI-1640 medium containing $10 \%$ fetal bovine serum (FBS) and $1 \%$ penicillin-streptomycin and incubated at $37^{\circ} \mathrm{C}$ and $5 \%$ $\mathrm{CO}_{2}$. Basic RPMI-1640 medium and FBS were obtained from Gibco (Grand Island, NY, USA). A glucose concentration of $5.0 \mathrm{mmol} / \mathrm{L}$ was used as the normal control group (NG), and $30 \mathrm{mmol} / \mathrm{L}$ as the $\mathrm{HG}$ group.

\section{Cell Transfection}

Transient transfection was performed with Lipofectamine 3000 (Lipo3000; Invitrogen, USA) transfection kits. MPC5 cells were cultured in $\mathrm{HG}$ and $\mathrm{NG}$ and transfected with silent-interfering negative-control RNA (siRNA-NC), siRNA-MALAT1, NC mimics, and miR-200c mimics. All transfection reagents were synthesized by Ruibo Biological Company (Guangzhou, China). SiRNA and mimic sequences are as follows: 
The si-MALAT1-1 sense: 5'-GUGAUGAAGGU AGCAGGCG-3' and antisense: 5'-CACUACUUCCAUCG UCCGC-3'. The si-MALAT1-2 sense: 5'- CAGGAUA AUCAGACCACCA and antisense: 5'- GUCCUAUUA GUCUGGUGGU-3'. The si-MALAT1-3 sense: 5'- GAAA

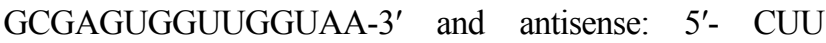
UCGCUCACCAACCAUU-3'. The miR-200c-mimics sense: 5'- UAAUACUGCCGGGUAAUGAUGGA-3' and antisense: 5'- UCCAUCAUUACCCGGCAGUAUUA-3'. The negativecontrol sense: 5'- UUUGUACUACACAAAAGUACUG-3' and antisense: 5'- CAGUACUUUUGUGUAGUACAAA-3'.

After 48 hours of transfection with Lipo3000, transfection effectiveness was evaluated in Western blots, qRTPCR, and other procedures.

\section{Western Blotting Assay}

Protein expression was analyzed by Western blotting. Total protein was extracted using precooled radioimmunoprecipitation assay buffer (RIPA buffer) with protease inhibitors (Beyotime, Shanghai, China), and the protein concentration was determined with bicinchoninic acid (BCA) assay kits (Beyotime, Shanghai, China). The proteins were separated by $10 \%$ sodium dodecyl sulfate polyacrylamide gel electrophoresis (SDS PAGE, at $100 \mathrm{~V}$ for 110 minutes and electrotransferred to polyvinylidene fluoride (PVDF) membranes (Millipore, Billerica, MA)). The membranes were blocked by immersion in 5\% skimmed milk for 1 hour at room temperature and then incubated with the primary antibodies overnight at $4{ }^{\circ} \mathrm{C}$. Membranes were incubated with horseradish peroxidase (HRP)conjugated secondary goat anti-mouse and anti-rabbit secondary antibodies for 1.5 hours at $37^{\circ} \mathrm{C}$, washed with Tris-buffered saline-tween, and binding was visualized by enhanced chemiluminescence (ECL; Bridgen, \#D046). The primary antibodies were GAPDH (1:2000, Cell Signaling Technology \#2118S), caspase-1 (1:1000, Bioss, bs-10442R), GSDMD (1:1000, Abcam, ab-219800), NLRP3 (1:1000, Bioss, bs-10021R), NRF2 (1:1000, Proteintech, 66504-1-Ig), HO-1 (1:1000, Cell Signaling Technology, \#43966). The secondary antibodies were goat anti-mouse IgG $(\mathrm{H}+\mathrm{L})(1: 4000$, ZSGB-BIO, ZB-2305) and goat antirabbit $\operatorname{IgG}(\mathrm{H}+\mathrm{L} ;$ 1:4000, ZSGB-BIO, ZB-2301). GAPDH was the internal control for quantifying protein levels by their gray values. Each assay was performed in triplicate.

\section{Quantitative Real-Time Polymerase Chain} Reaction (qRT-PCR)

Total RNA was extracted with RNA Simple Total RNA Kits (China Tiangen \#DP419) and was reverse-transcribed into cDNA with Thermo Fisher Revert Aid First Stand cDNA Synthesis Kits (Invitrogen, \#K1622, USA) or Mir-XTM miRNA First-Strand Synthesis Kits (US Clontech Laboratories, NOs. 638313). SYBR Premix Ex Taq II (Japan TAKARA) was used to perform the qRT-PCR assays. Primer sequences used in the qPCR assays are as follows:

The Caspase-1 forward: 5'-ACAAGGCACGGGACCT ATG-3' and reverse: 5'- TCCCAGTCAGTCCTGG AAATG-3'. The GSDMD forward: 5'- CCAGCAT GGAAGCCTTAGAG-3' and reverse: 5'- CAGAGT CGAGCACCAGACAC-3'. The NLRP3 forward: 5'GTGTGGATCTTTGCTGCGAT-3' and reverse: 5'ACAAATGGAGATGCGGGAGA-3'. The NRF2 forward: 5'- CTTTAGTCAGCGACAGAAGGAC-3' and reverse: 5'- AGGCATCTTGTTTGGGAATGTG-3'. The MALAT1 forward: 5'- ACGCAGGTGTGGCTTTCCAT-3' and reverse: 5'- GTCCTCCCTCACCACAATGG-3'. The miR-200c forward: 5'- GCCCGCTAATACTGCCGGGT AAT-3' and reverse: 5'- GTGCAGGGTCCGAGGT-3'. The GAPDH forward: 5'- TGGCCTTCCGTGT TCCTAC-3' and reverse: 5'- GAGTTGCTGTTGAA GTCGCA-3'. The U6 forward: 5'- CTCGCTTCGGC AGCACA-3' and reverse: 5'-AACGCTTCACGAAT TTGCGT-3'.

Glyceraldehyde 3-phosphate dehydrogenase (GAPDH) or U6 were the internal references, and the contrast CT $\left(2^{-\Delta \Delta \mathrm{CT}}\right)$ method was used to calculate relative mRNA expression. Each assay was repeated in triplicate.

\section{Immunofluorescence}

The expression of caspase-1, NRF2 was evaluated by immunofluorescence. Following experimental treatments such as HG or transfection and culture for 48 hours, cells were fixed in precooled $4 \%$ paraformaldehyde for 20 minutes, washed three times with PBS, blocked with 5\% bovine serum albumin for 1 hour, and incubated with caspase-1 (1:500), and NRF2 (1:500) antibodies at $4^{\circ} \mathrm{C}$ overnight. The next day, the cells were incubated with 488-conjugated AlexaFluor antirabbit IgG and 594-conjugated AlexaFluor 594 anti-mouse $\operatorname{IgG}(1: 500$, ZSGB-BIO) for 2 hours and counterstained with DAPI with anti-fluorescence quenching. The cells were observed and photographed with an Olympus inverted fluorescence microscope. 


\section{CCK-8 Assay}

CCK-8 kits (Dojindo Molecular Technologies, Japan) were used to assay the inhibition of podocyte pyroptosis in MPC-5 cells after AT treatment. AT concentrations of 1 , $2.5,5,10,15$, or $20 \mu \mathrm{mol} / \mathrm{L}$ were added after HG treatment to 96 -well plates that contained 1000 cells/well. Five replicate wells were used for each AT sample. After culturing for $48 \mathrm{~h}, 10 \mu \mathrm{L}$ CCK-8 was added to each well and incubated for 1-4 h. Absorbance was read at $450 \mathrm{~nm}$ with a microplate reader (iMarkLMicroplate Reader; Bio-Rad, USA). The most effective AT concentration was determined from optical density (OD) curves. The assays were repeated in triplicate.

\section{Detection of MDA, SOD and GSH}

\section{Activity}

After 48 hours of HG treatment or transfection, cells were cultured in RPMI-1640 and washed with PBS. Then, following to the kit manufacturer's instructions, the supernatant GSH (Nanjing Jianshe, China, A006-2-1), SOD (built in Nanjing, China, A001-3) and MDA (built in Nanjing, China, A003-2) level. Each experiment was repeated in triplicate.

\section{Cell Pyroptosis Detected by Flow Cytometry Assays}

Cells were treated with HG, transfection, or AT for $48 \mathrm{~h}$, collected and prepared following the kit manufacturer's instructions. After washing twice in cold PBS, cells were suspended in $1 \times$ binding buffer (BD Biosciences, San Diego, California). The suspensions were kept in the dark for addition of $5 \mu \mathrm{L}$ fluorescein isothiocyanate (FITC) Annexin V (BD Biosciences) for $10 \mathrm{~min}$ and $5 \mu \mathrm{L}$ of propidium iodide (PI, BD Biosciences). The cells were gently agitated and incubated at room temperature for $15 \mathrm{~min}$ in the dark before adding $400 \mu \mathrm{L} 1 \times$ binding buffer. Pyroptosis was assayed by flow cytometry (FACSCalibur, BD Biosciences). Each assay was repeated in triplicate.

\section{Statistical Analysis}

SPSS 22.0 (IBM Corp. Armonk, NY, USA) was used to analyze the data. The independent-sample $t$-test was used to determine the significance of between-group differences. $P$-values of $<0.05$ indicated statistically significant differences. GraphPad Prism version 8.0 (GraphPad, IBM, USA) was used to draw the graphs.

\section{Results}

\section{Effects of Culturing Time and Glucose Concentration on Pyroptosis and OS}

Pyroptosis of MPC- 5 cells was assayed by the expression caspase- 1 after 12, 24, 36, and $48 \mathrm{~h}$ and was the highest after $48 \mathrm{~h}$ of culture in HG (Figure 1A and B). At glucose concentrations of $10,20,30$, and $40 \mathrm{mmol} / \mathrm{L}$ in 48 -hour cultures, pyroptosis was the highest at $30 \mathrm{mmol}$ (Figure 1C and D). Western blotting assays of caspase-1, GSDMD, NLRP3, NRF2 and HO-1 protein expression (Figure 1E and F) and qRT-PCR assays of caspase-1, GSDMD, NLRP3, and NRF2 mRNA expression (Figure 1G) showed that HG upregulated caspase-1, GSDMD and NLRP3, and downregulated NRF2 expression, consistent with HGpromotion of cell pyroptosis and increased OS. Results of the immunofluorescence assays of caspase- 1 and NRF2 expression after 24 hours of HG treatment were consistent with those obtained by Western blotting and qPCR (Figure 1H). These results showed that HG upregulated caspase-1, GSDMD, NLRP3 and downregulated NRF2, suggesting that HG promoted pyroptosis and OS induced by HG.

\section{Optimal Concentration of AT}

AT has a protective effect on MPC-5 cells, but a high dose may cause cell death. To determine the optimal dose of AT, we used the CCK- 8 assay to evaluate pyroptosis inhibition with AT doses of 1, 2.5, 5, 10 15, and $20 \mu \mathrm{mol} / \mathrm{L}$ and glucose concentration of $30 \mathrm{mmol} / \mathrm{L}$. The inhibition rate was the smallest at $2.5 \mu \mathrm{mol} / \mathrm{L}$ (Figure 2A). We also compared the mRNA expression of caspase- 1 and NLRP3 at HG condition of $30 \mathrm{mmol} / \mathrm{L}$ with AT concentrations of 1 and $2.5 \mu \mathrm{mol} / \mathrm{L}$ by qRTPCR. The optimal AT concentration was $2.5 \mu \mathrm{mol} / \mathrm{L}$ (Figure 2B). Differences in caspase-1, GSDMD, NLRP3, NRF2 mRNA expression and protein level in the NG, HG, and AT-treated groups indicated that HG significantly increased pyroptosis and OS and that the effects of HG were reversed by AT (Figure 2C-E). Flow cytometry confirmed that AT alleviated the pyroptosis of MPC-5 cells (Figure 2F). And as shown in Figure 2G, the GSH and SOD levels in the HG group decreased, and the MDA level increased, and the trend was reversed after the administration of AT. The immunofluorescence assay results of caspase-1 and NRF2 protein expression in the NG, HG and HG plus AT groups are shown in Figure $2 \mathrm{H}$. 

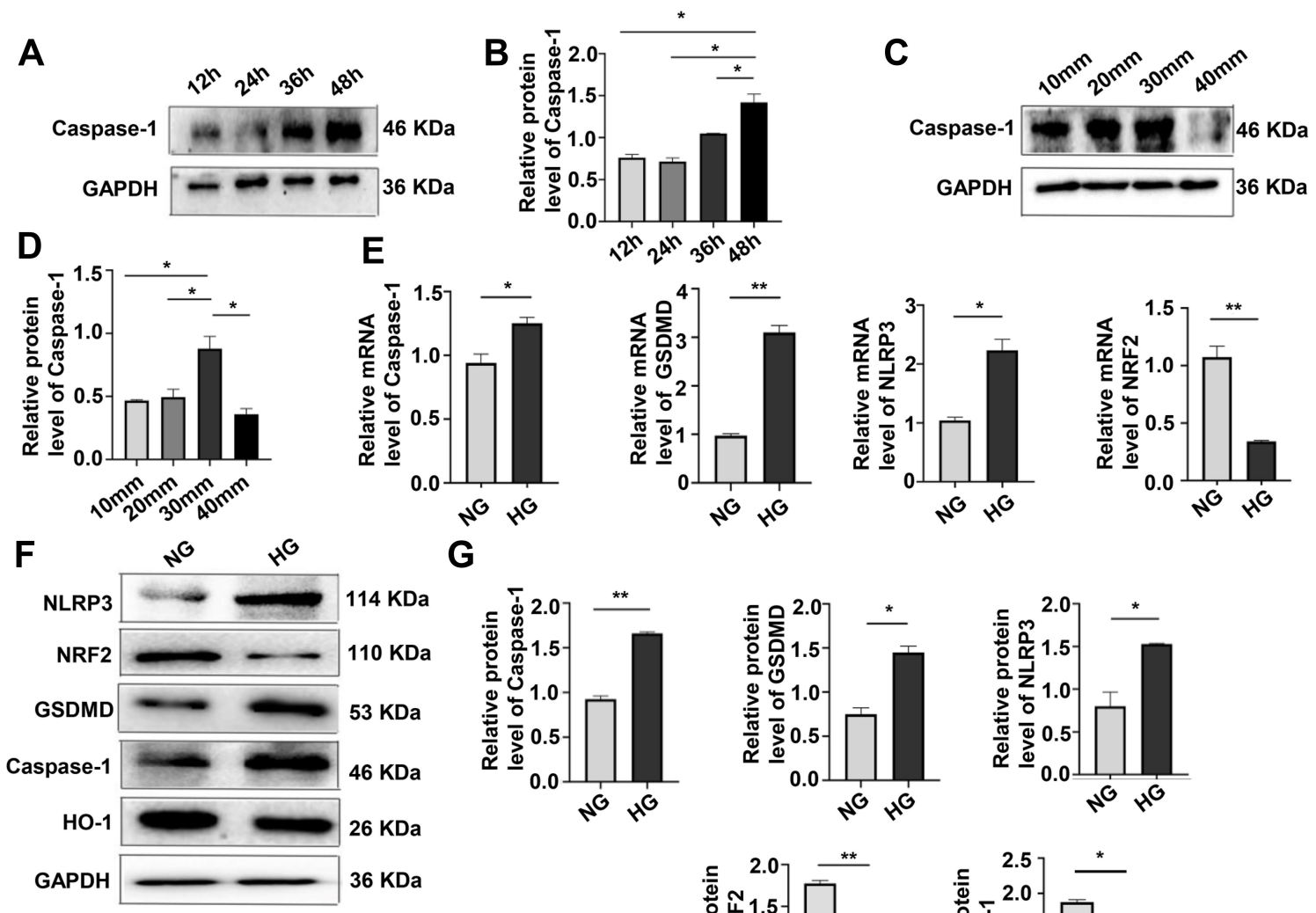

D

F

H
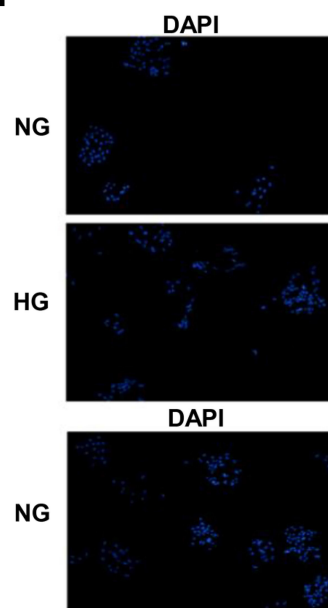

HG

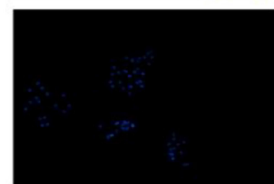

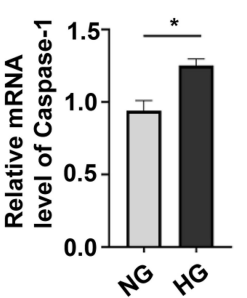

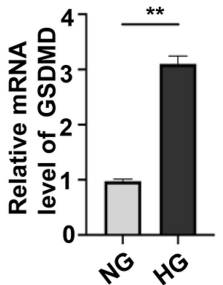

G
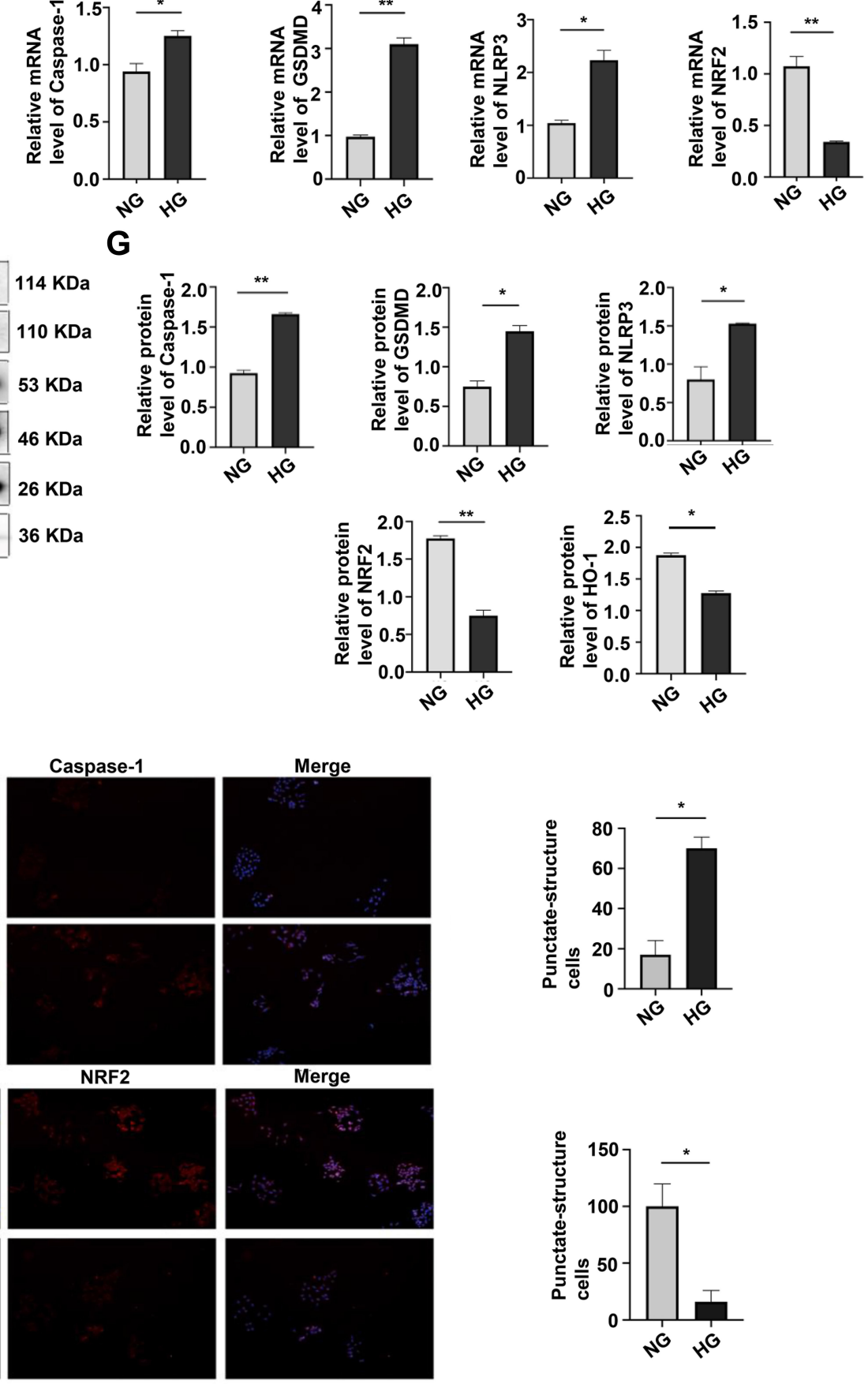

Figure I Effects of culturing time and glucose concentration on pyroptosis and OS. (A and B) Western blotting of caspase-I expression (pyroptosis) after I2, 24, 36, and 48 $\mathrm{h}$ of culture showed the highest level was at $48 \mathrm{~h}$. $* P<0.05$. (C and D) Western blotting of caspase-I expression after $48 \mathrm{~h}$ of culture at glucose concentrations of I0, 20, 30, $40 \mathrm{mmol} / \mathrm{L}$, showing the highest level at $30 \mathrm{mmol} / \mathrm{L}$. *P $<0.05$. (E) mRNA expression of caspase-I, GSDMD, NLRP3 and NRF2 at NG and HG by qRT-PCR. *P <0.05, $* * P<0.0$ I. (F and $\mathbf{G})$ Levels of caspase-I, GSDMD, NLRP3, NRF2, HO-I, in HG treatment by Western blotting. $* P<0.05$, $* * P<0.01$. (H) Immunofluorescence assays of caspase-I, and NRF2 were performed following the induction of HG. $* P<0.05$. 

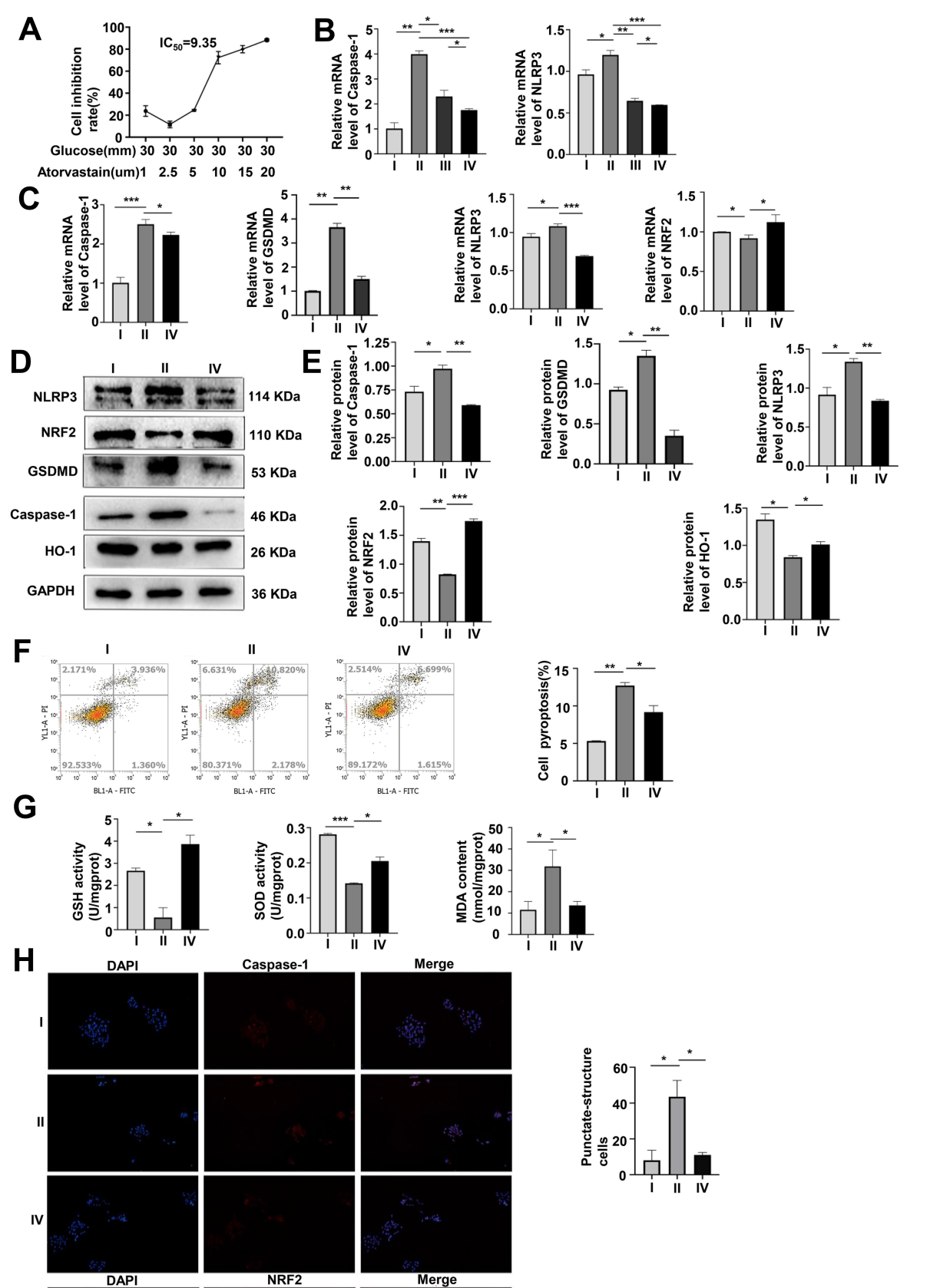


\section{The Knockdown of MALATI Protected MPC-5 Cells from Pyroptosis and OS Induced by HG}

As shown in Figure 3A and B, the interference of HG led to the increase in MALAT1 and miR-200c-3p in MPC-5 cells that were reversed by AT.

We divided MPC-5 cells into five groups: $\mathrm{HG}+$ control (only lipo3000), HG + NC siRNA, HG + MALAT1-1 siRNA, HG + MALAT1-2 siRNA, and HG + MALAT1-3 siRNA. Transfection efficiency was the best with the MALAT1-3 siRNA fragment (Figure 3C). Protein and mRNA expression of caspase-1, GSDMD, NLRP3, NRF2 and miR-200c were assayed after transfection with MALAT1 and showed that both pyroptosis and OS had decreased compared with controls (Figure 3D-F). The results were consistent with those obtained by the flow cytometry and immunofluorescence (Figure 3G and I). The levels of GSH, SOD and MDA also verified this trend (Figure 3H).

\section{Interference of miR-200c-3p Suppressed the Pyroptosis and OS in HG-Induced MPC-5 Cells}

As shown in Figure 4A, pyroptosis, OS and the expression of MALAT1 increased with the overexpression of miR$200 \mathrm{c}$ at normal glucose concentration of $5 \mathrm{mmol} / \mathrm{L}$. That trend was confirmed by the Western blotting assay results (Figure 4B and C), and results of flow cytometry verified that both levels of OS and pyroptosis increased with the overexpression of miR-200c (Figure 4D). Upregulation of miR-200c resulted in the decreased levels of GSH and SOD and increased levels of MDA (Figure 4E). The immunofluorescence results were consistent with those obtained with the other assays (Figure 4F).

\section{Overexpression of NRF2 Had}

a Protective Effect on Podocytes Induced by HG, Unable to Influence the Level of MALATI and miR-200c

In order to explore the protective effect of NRF2 on podocytes stimulated by HG, we divided MPC- 5 cells into four groups: $\mathrm{HG}+$ Control, $\mathrm{HG}+$ pcDNA-Control, HG+pcDNA-NRF2 and HG+pcDNA-NRF2+AT. The pyroptosis and OS levels of the NRF2 overexpression were tested by qRT-PCR and Western blotting assays. It was found that after the overexpression of NRF2, the levels of both significantly decreased, and the application of AT further alleviated the levels of both
(Figure 5A-C). The results of GSH, SOD and MDA also confirmed this trend (Figure 5D). In Figure 5A, we found that after overexpressing NRF2, there was no evident difference in the expression of MALAT1 and miR-200c.

For the purpose of further proving the relationships among NRF2, MALAT1 and miR-200c, we set up HG + miR-200c mimics $+\mathrm{Si}-\mathrm{NC}$ and $\mathrm{HG}+$ miR-200c mimics $+\mathrm{Si}-$ MALAT1 groups and detected the levels of MALAT1 and NRF2 by qRT-PCR. We found that the level of MALAT1 decreased and that of NRF2 did not change significantly (Figure 5E). We also set up HG + pcDNA-MALAT1 + inhibitor control and HG + pcDNA-MALAT1 + miR-200c inhibitor groups, and tested the levels of miR-200c and NRF2 by qRT-PCR. It was found that the level of miR-200c decreased, and the expression of NRF2 did not change significantly (Figure 5F).

\section{Pyroptosis and OS Induced by HG are Reversed by AT}

The role of AT in pyroptosis and OS was investigated by Western blotting in the HG + control, HG + NC siRNA, HG + MALAT1 siRNA, HG + MALAT1 siRNA + AT and NG + control, $\mathrm{NG}+\mathrm{NC}$ siRNA mimics, $\mathrm{NG}+$ miR-200c mimics, $\mathrm{NG}+$ miR-200c-mimics + AT groups. Both pyroptosis and OS decreased significantly after the treatment of AT (Figure 6AD). Results of flow cytometry confirmed that of Western blotting (Figure 6E and F). The levels of GSH, SOD and MDA are consistent with results above (Figure 6G and $\mathrm{H}$ ).

\section{Discussion}

DM is one of the most prevalent metabolic diseases worldwide. It is characterized by hyperglycemia and multiple organ complications. DM accelerates cell senescence, and high blood-sugar levels are associated with the occurrence of cardiovascular and renal diseases. ${ }^{29,30}$ As one of the chronic complications, DN is a common risk factor, independent of kidney and cardiovascular diseases. ${ }^{31,32}$

Pyroptosis is a lytic form of cell death characterized by swelling and rupture with release of the cell contents. Pyroptosis is mediated by Gasdermin-family proteins including inflammatory factors such as IL-18, IL- $1 \beta,{ }^{33}$ whose classical pathway depends on the release of caspase-1 promoted by pro-caspase-1 and pattern recognition receptors (eg, NLRP1, NLRP3, NLRC4, AIM2 and pyrin), with formation of inflammasomes. GSDMD belongs to the Gasdermin family. Pro-caspase-1 is activated by apoptosis-associated speck-like protein containing a caspase 


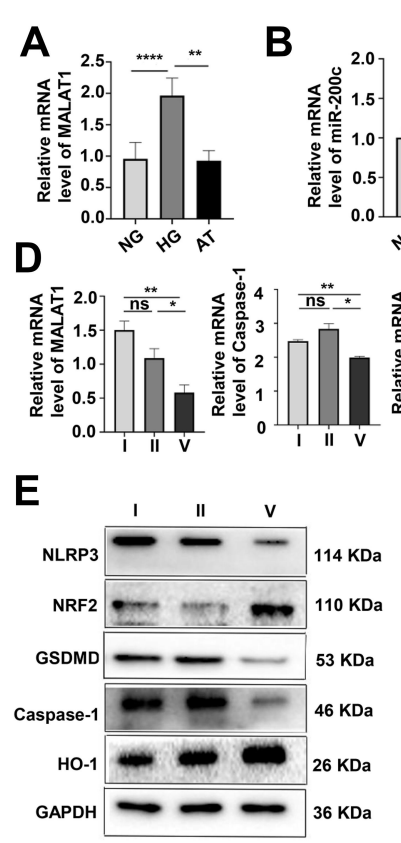

$\mathbf{G}$
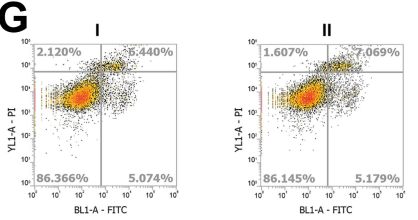
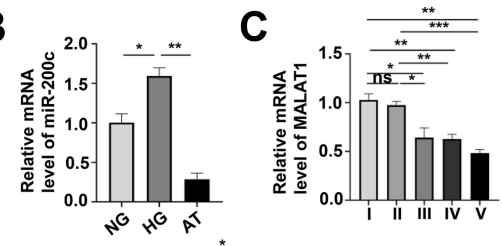
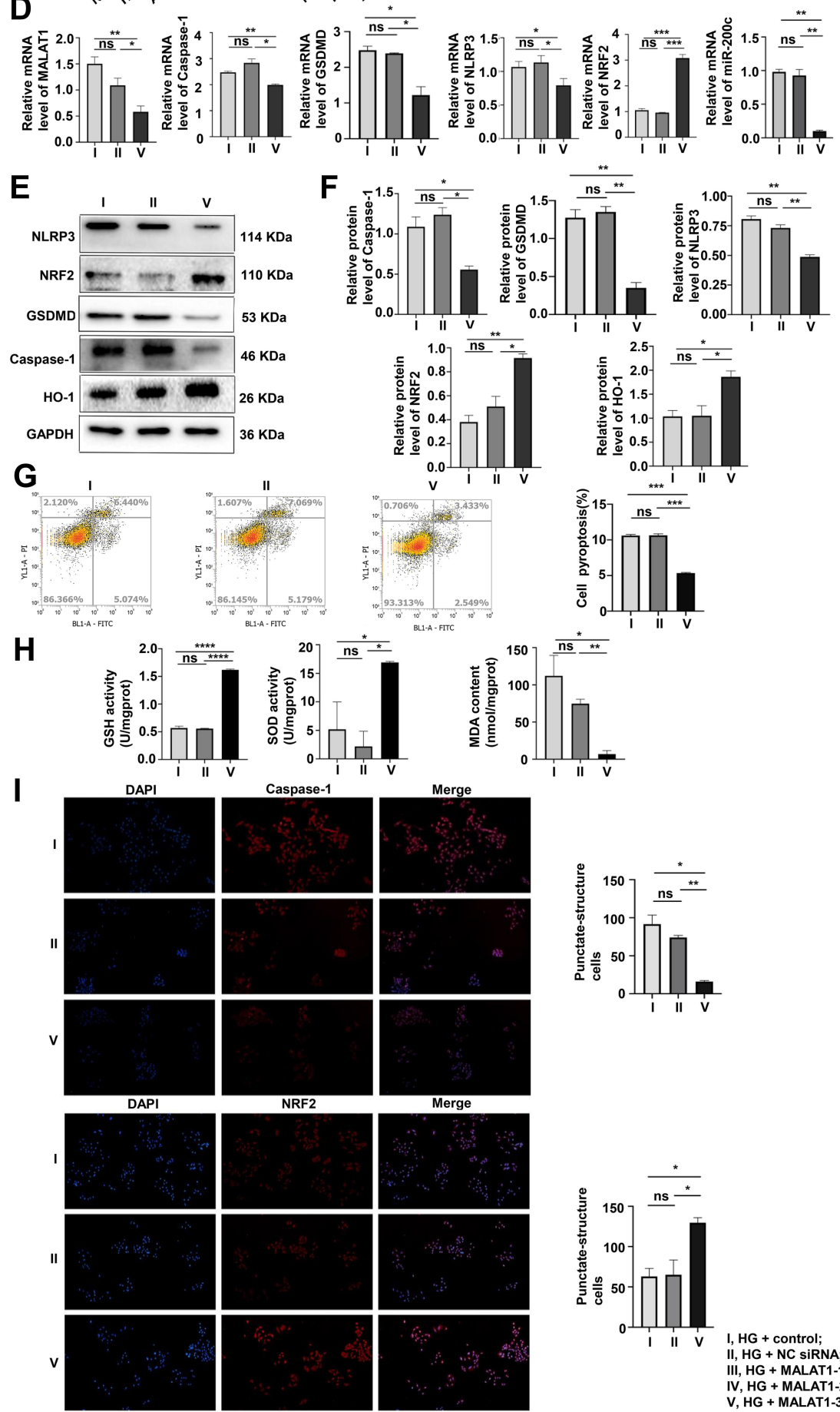

NRF2
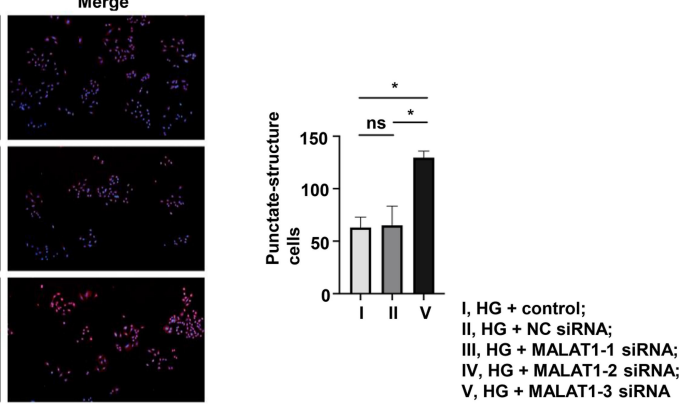

Figure 3 The knockdown of MALATI inhibited pyroptosis and OS induced by HG. (A and B) MALATI and miR-200c mRNA were assayed by qRT-PCR. The results supported the transfection investigation. $* P<0.05$, $* * P<0.01$, $* * * * P<0.000$ I. (C) After transfection with MALATI-I, 2 , and 3 siRNA, qPCR assay of MALATI mRNA expression identified the fragment with the best transfection efficiency. $* P<0.05$, $* * P<0.01$, $* * * P<0.001$, ns, non significant. (D-F) Pyroptosis and OS were evaluated after the knockdown of MALATI by qPCR and Western blotting. $* P<0.05$, $* * P<0.01$, $* * * P<0.00 I$, ns, non significant. (G) Pyroptosis after transfection with MALATI was analyzed by flow cytometry. $* * * P<0.00 \mathrm{I}$. (H) GSH, SOD, and MDA levels were assayed after the transfection of MALATI. $* P<0.05$, $* * P<0.0 \mathrm{I}$, $* * * * P<0.000 \mathrm{I}$, ns, non significant. (I) Levels of caspase-I, and NRF2 in cells transfected with MALATI were assayed by immunofluorescence. I, HG + control; II, HG + NC siRNA; III, HG + MALATI-I siRNA; IV, HG + MALATI-2 siRNA; V, HG + MALATI-3 siRNA. *P<0.05, **P<0.0I, ns, non significant. 

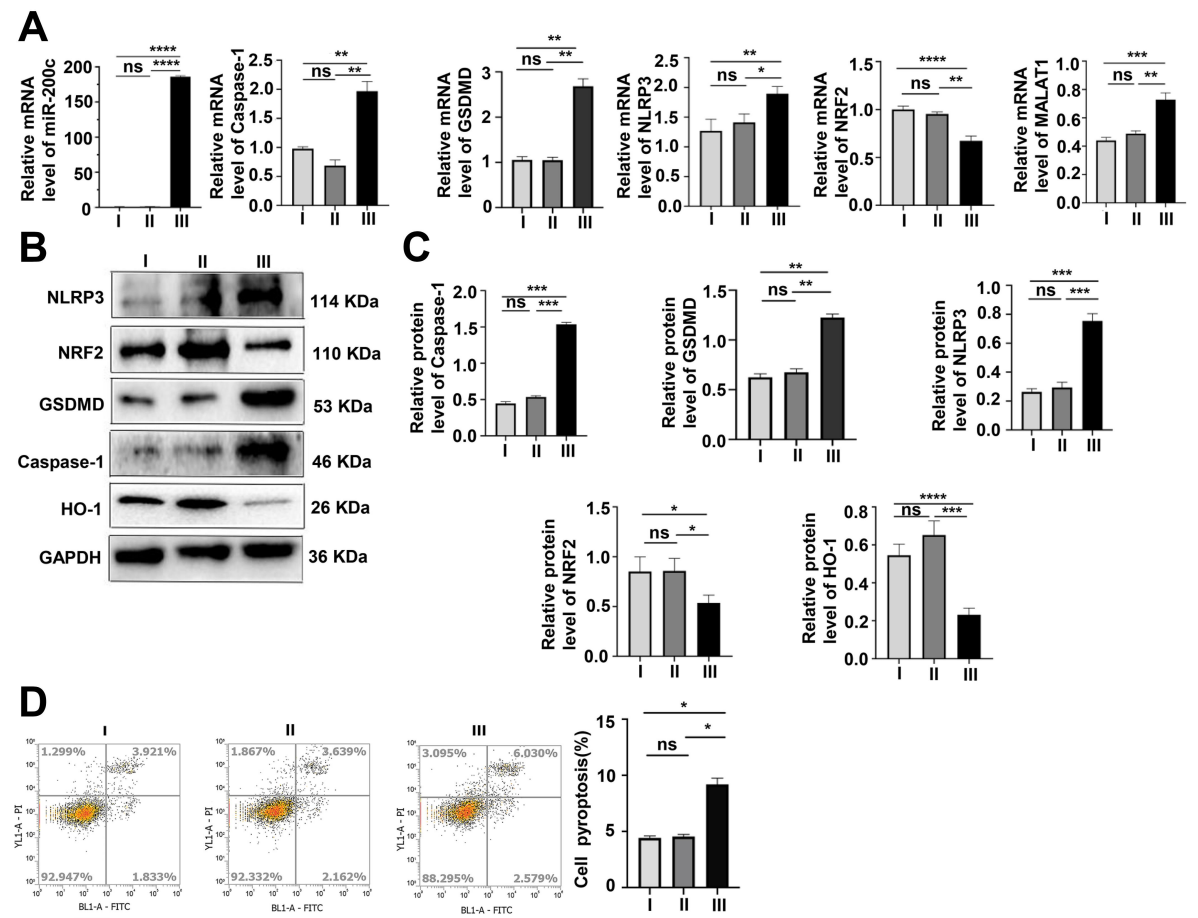

E
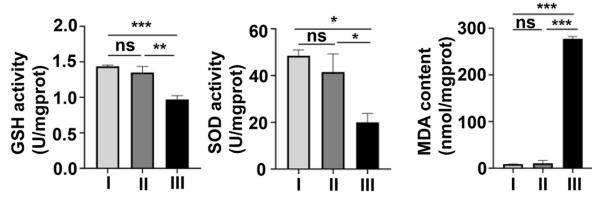

$\mathbf{F}$
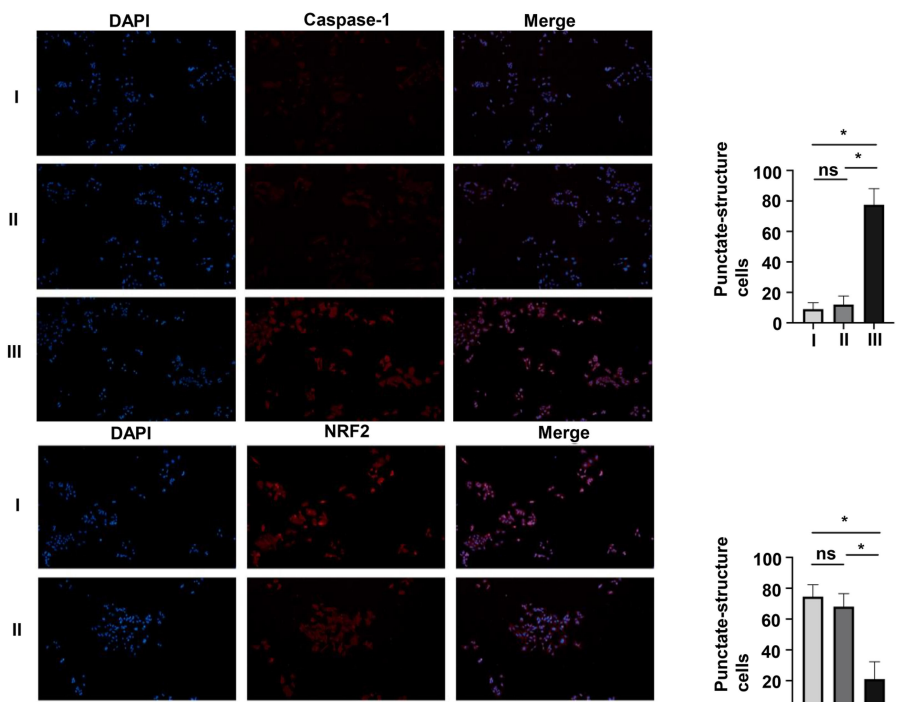

III
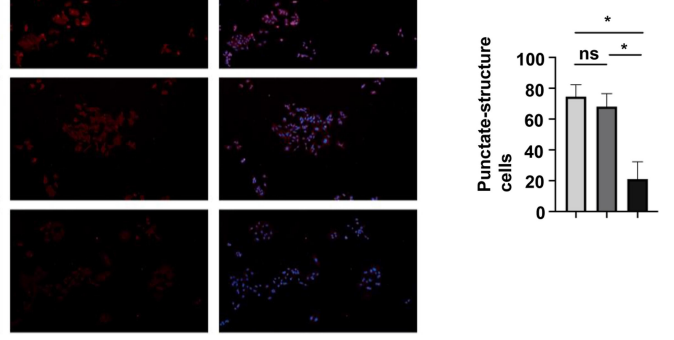

Figure 4 Interference of miR-200c reversed pyroptosis and OS in MPC-5 cells. (A-C) As shown by qRT-PCR and Western blotting results, the levels of pyroptosis and OS were detected. $* P<0.05, * * P<0.01$, $* * * P<0.001$, $* * * * P<0.000 \mathrm{I}$, ns, non significant. (D) After the interference of miR-200c, the pyroptosis rate was analyzed by flow cytometry. $* P<0.05$, ns, non significant. (E) The results of GSH, SOD and MDA levels were consistent with others above. $* P<0.05$, $* * P<0.0$ I, $* * * P<0.00 \mathrm{I}$, ns, non significant. (F) Levels of caspase-I, and NRF2 in cells were assayed by immunofluorescence. I, NG + control; II, NG + mimics-NC; III, NG + miR-200c mimics. *P <0.05, ns, non significant. 
A
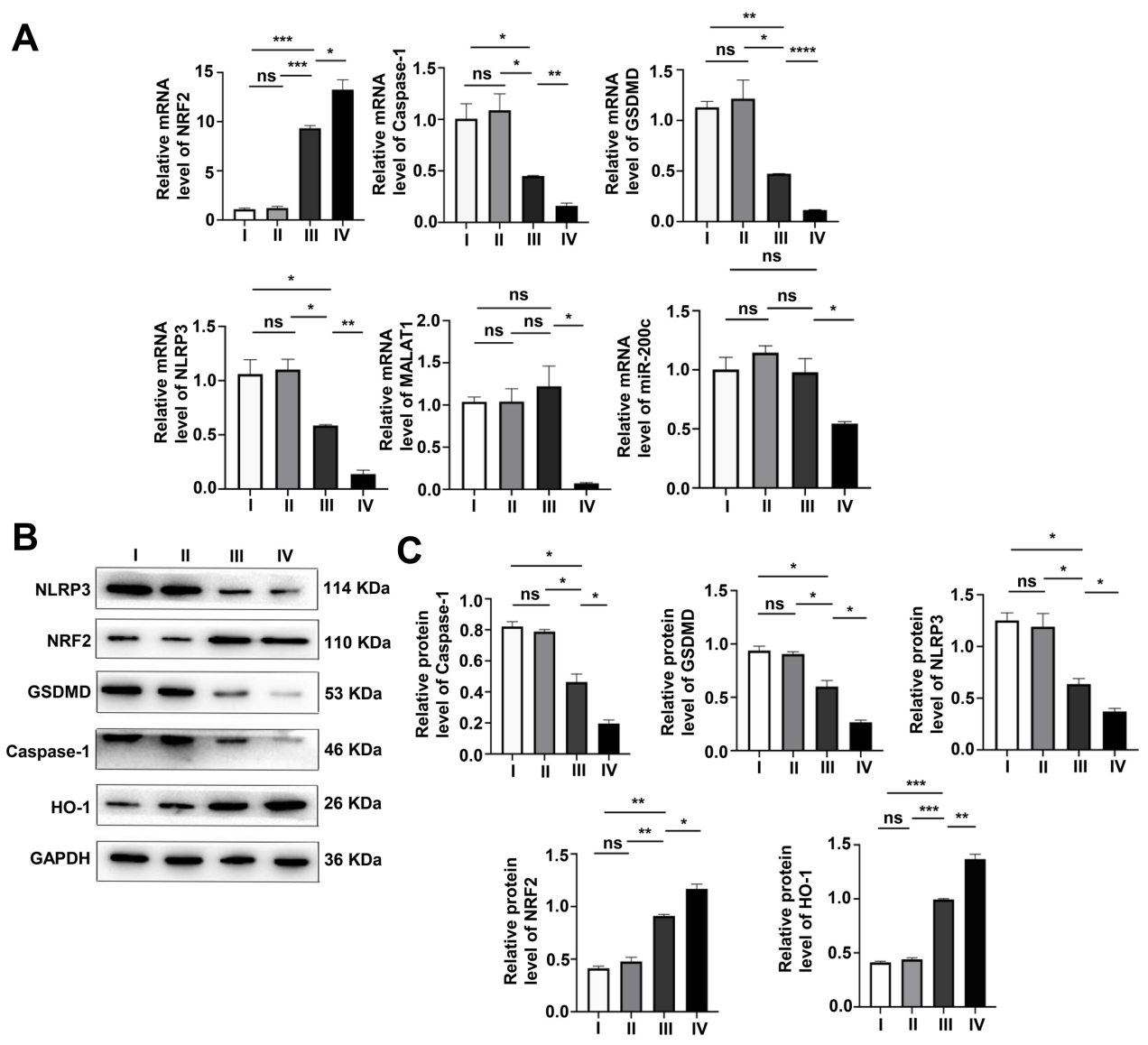

D

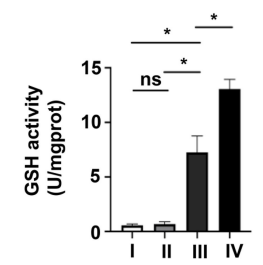

E

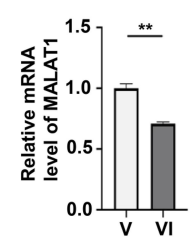

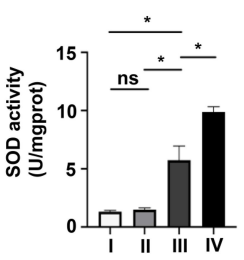

$\mathbf{F}$
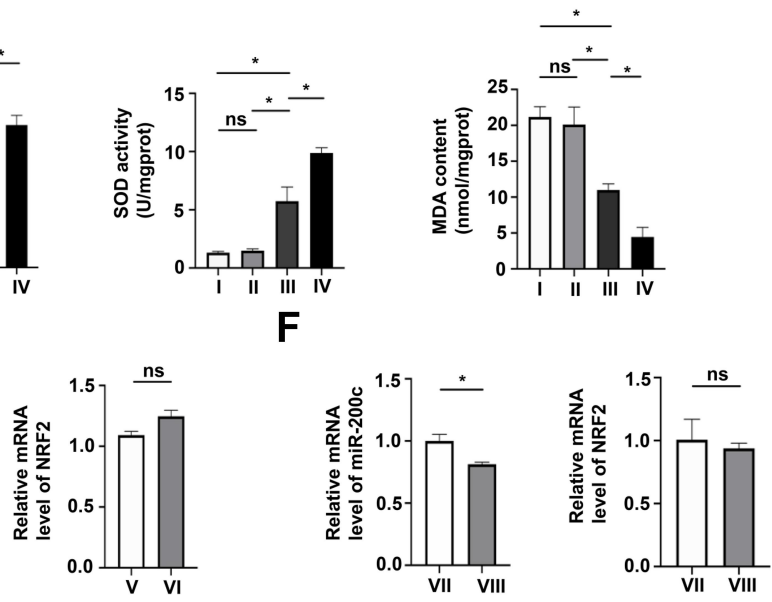

I, HG+ control;

II, HG + pcNDA-NC;

III, HG + pcNDA-NRF2

IV, $H G$ + pcNDA-NRF2 + AT

V, HG + miR-200c mimics + Si-NC;

VI, HG + miR-200c mimics + Si-MALAT1;

VII, HG + pcNDA-MALAT1 + inhibitor-NC;

VIII, HG + pcNDA-MALAT1 + miR-200c inhibitor

Figure 5 The overexpression of NRF2 alleviated pyroptosis and OS, unable to interfere with the expression of MALATI and miR-200c. (A-C) The pyroptosis and OS levels of the NRF2 overexpression group were determined by WB and qRT-PCR. *P<0.05, **P<0.01, ***P<0.00I, $* * * * P<0.0001$, ns, non significant. (D) GSH, SOD and MDA levels. $* P<0.05$, ns, non significant. (E and F) qRT-PCR was used to verify that NRF2 is a downstream factor cannot, incapable of interfering with the levels of MALATI and miR200c. *P<0.05, **P<0.0I, ns, non significant. I, HG+ control; II, HG + pcNDA-NC; III, HG + pcNDA-NRF2; IV, HG + pcNDA-NRF2 + AT; V, HG + miR-200c mimics + Si-NC; VI, HG + miR-200c mimics + Si-MALATI; VII, HG + pcNDA-MALATI+ inhibitor-NC;VIII, HG + pcNDA-MALATI+ miR-200c inhibitor. 

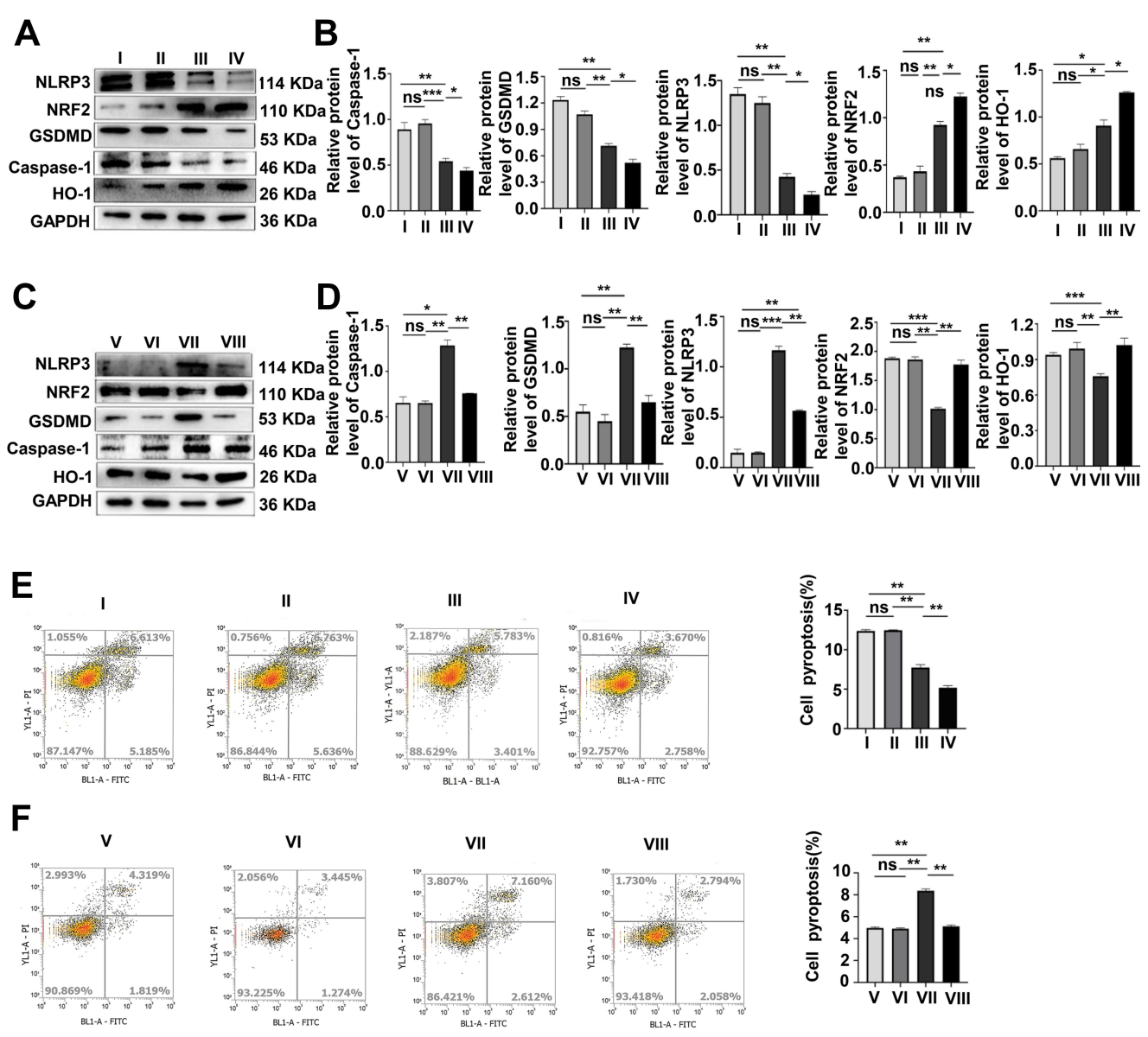

G
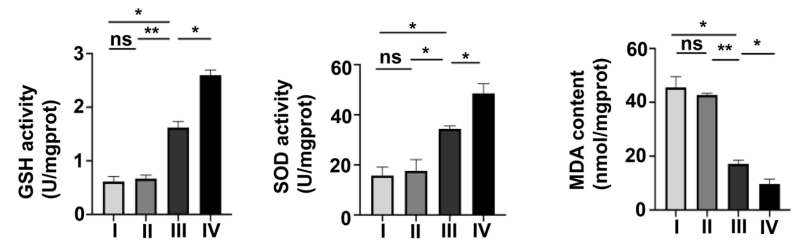

H
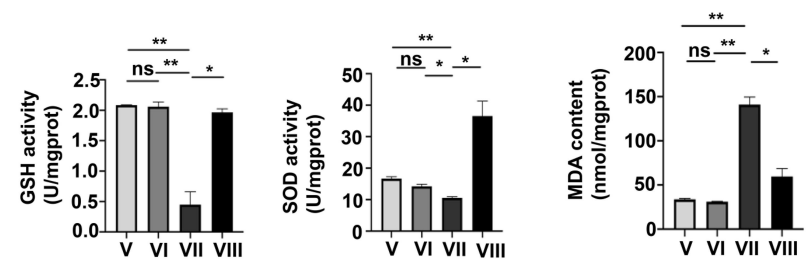

I, HG + control;

II, HG + NC siRNA

III: HG + MALAT1 SIRNA;

IV, HG + MALAT1siRNA + AT;

V, NG + control;

VI, NG + mimics-NC:

VII, NG + miR-200c mimics;

VIII, NG + miR-200c mimics;
+ miR-200c mimics + AT

Figure 6 AT acts to suppress pyroptosis and OS via MALATI/miR-200c/NRF2 activity. (A-D) The effects of AT on pyroptosis and OS of the AT group after MALATI knockdown and miR-200c overexpression were assayed by Western blotting. ${ }^{*} P<0.05$, $* * P<0.0$, $* * * P<0.00 \mathrm{I}$, ns, non significant. (E and $\left.\mathbf{F}\right)$ Flow cytometry, (G and $\mathbf{H}$ ) GSH, SOD and MDA levels. **P <0.0I, ns, non significant. I, HG + control; II, HG + NC siRNA; III, HG + MALATI siRNA; IV, HG + MALATI siRNA + AT; V, NG + control; VI, NG + mimics-NC; VII, NG + miR-200c mimics; VIII, NG + miR-200c mimics + AT. 
recruitment domain (ASC). Caspase-1 induces the release of IL-1 $\beta$ and IL-18. The release of intracellular materials and expanded inflammatory response mediates cell and tissue damage. ${ }^{34}$ Pyroptosis is known to occur in DN. ${ }^{35-40}$ OS can also lead to the occurrence of pyroptosis, with production of reactive oxygen species activating NLRP3 inflammasomes, indirect activation of caspase-1, and promotion of IL-1 $\beta$ and IL-18 production that lead to inflammation and pyroptosis. ${ }^{13,41,42}$

It has been reported that $\mathrm{HG}$ can affect the expression of NRF2. ${ }^{43,44}$ Mathur et $\mathrm{al}^{45}$ reported that increased OS in response to HG influenced signal pathways inhibiting the NRF2 transcription resulting in oxidative damage, disorders of glucose metabolism, and cell death. Diao et $\mathrm{al}^{46}$ reported that arginine methylation transferase 5 (PRMT5) activated NRF2/HO-1 to inhibit OS and pyroptosis, thus leading to the remission of renal damage. Mu et $\mathrm{al}^{47}$ found that that piceatannol (PIC) decreased OS and pyroptosis in atherosclerosis (AS) via miR-200a/NRF2/GSDMD signaling. The available evidence thus indicates that NRF2 is involved in the modulation of pyroptosis and OS.

LncRNAs are long noncoding RNAs with a length of more than 200 nucleotides that are novel biomarkers and therapeutic target in some human diseases. ${ }^{48,49}$ Recent studies have reported an association between lncRNAs and the occurrence and development of $\mathrm{DN}^{50-53}$ MALAT1, also known as noncoding nuclear-enriched abundant transcript 2 (NET2), is an lncRNA that is widely expressed in human tissues and highly conserved in 33 species of mammals. It has also been reported that MALAT1 plays an important role in DM and its complications. ${ }^{54,55} \mathrm{Li}$ et $\mathrm{al}^{35}$ found the expression of MALAT1 increased and that of miR-23c decreased in rats with streptozotocin-induced $\mathrm{DM}$ and HG-induced HK-2 renal tubular epithelial cells. Downregulation of MALAT1 expression or upregulating miR-23c expression might inhibit pyroptosis, with decreased expression of NLRP3, caspase-1 and IL-1 $\beta$, suggesting that downregulation of MALAT1 inhibits pyroptosis induced by HG. MiRNAs are noncoding RNAs that regulate gene expression, for example by inhibiting lncRNAs near target genes in the cell nucleus. ${ }^{55}$ The mechanisms of miRNA regulation of target genes need further investigation. MiR-200c, a member of miR-200 family, is involved in the regulation of OS. Carlomosti et $\mathrm{al}^{18}$ found that it was upregulated in endothelial cells in vitro and was associated with increased OS damage. Cortez et $\mathrm{al}^{56}$ reported that miR-200c had antitumor effects that were mediated by targeting NRF2, but $\mathrm{Li}$ et al found that the combination of miR-200c and MALAT1 was associated with increased migration and invasiveness of endometrial cancer. ${ }^{19}$

In this study, the expression of caspase-1 and GSDMD, key promoters of pyroptosis, and NRF2 and NLRP3, which participate in the process of OS, all changed in podocytes cultured in HG condition, with corresponding surging of pyroptosis and OS. The expression of HO-1, the only downstream protein of NRF2, also decreased. We found that AT partially alleviated the changes in OS and pyroptosis induced by HG. HG also upregulated levels of MALAT1 and miR-200c. Transfection studies found that the knockdown of MALAT1 alleviated pyroptosis and OS in cultured podocytes in response to HG. Overexpression of miR-200c in transfected cells cultured at normal glucose concentrations promoted cell pyroptosis and OS. However, the upregulation of NRF2 alleviated pyroptosis and OS induced by HG. Furthermore, we found that after down-regulating MALAT1, the level of miR-200c decreased and the level of NRF2 increased; after overexpression of miR-200c, the level of MALAT1 increased, while the level of NRF2 decreased; after overexpression of NRF2, the levels of MALAT1 and miR-200c remained unchanged. In addition, we overexpressed miR-200c on the basis of knocking down MALAT1, and the level of NRF2 did not change, while knocking down miR-200c after upregulating MALAT1 did not change the level of NRF2. These confirmed that after down-regulation of MALAT1, even if miR-200c was overexpressed, the level of NRF2 did not change. And after up-regulating MALAT1, without the participation of miR-200c, the expression of NRF2 did not change significantly. These hinted that after upregulating MALAT1, the expression of NRF2 did not change without the participation of miR-200c. These above suggested that MALAT1 regulates the expression of NRF2 through miR-200c, while NRF2 as a downstream factor cannot interfere with the expression of MALAT1 and miR-200c. Besides, AT after transfection partially alleviated OS and pyroptosis.

Based on the study results, we believe that podocytes undergo pyroptosis and OS due to HG. If glucose metabolism is disturbed, interaction of MALAT1, miR-200c, and NRF2 takes part in the regulation of the pathogenesis of OS-related podocyte pyroptosis. AT can reduce renal OS and pyroptosis under HG conditions, which may be mediated by the MALAT1/miR-200c/NRF2 axis. 
The innovation of our research is the first study of atorvastatin regulating high glucose-induced mouse podocyte pyroptosis and oxidative stress through MALAT1/ miR-200c/NRF2 in order to explore the pathogenesis of DN, however, some shortcomings still exist. First of all, our research pathway is currently limited to cell experiments, which is not involved in animal models and clinical cases. Secondly, our research did not focus on the relationships between high glucose-induced podocyte oxidative stress and pyroptosis. For example, we did not interfere with the key factor of oxidative stress, NLRP3, to detect the effects on pyroptosis and this regulatory axis. This may become a deficiency in our experimental design. In fact, the occurrence and development of pyroptosis, oxidative stress, LncRNA, miRNA and DN have constituted a huge regulatory network. So as to explain the specific pathogenesis of DN, more research is needed to explore the genetic regulatory network of $\mathrm{DN}$.

In conclusion, we found that MALAT1/miR-200c/ NRF2 was involved in the regulation of pyroptosis and OS in podocytes. HG promoted pyroptosis, and OS and AT alleviated those effects via the same axis. Studies of LncRNAs may confirm their involvement in disease mechanisms. MALAT1 and NRF2 play an important role in the pyroptosis that occurred in DN and increased understanding of significance for the diagnosis and treatment of DN.

\section{Acknowledgments}

This project was supported by a grant from the National Natural Science Foundation of China (No. 81860156 and No. 81860148). We thank International Science Editing (http://www.internationalscienceediting.com) for editing this manuscript.

\section{Author Contributions}

Suxian Zhou, Xiaoyun He and Yi Zuo conceived and designed this study. Yi Zuo conducted the main experiments. Yi Zuo and Li Chen both participated in data analyses and manuscript writing. All authors made a significant contribution to the work reported, whether that is in the conception, study design, execution, acquisition of data, analysis and interpretation, or in all these areas; took part in drafting, revising or critically reviewing the article; gave final approval of the version to be published; have agreed on the journal to which the article has been submitted; and agree to be accountable for all aspects of the work.

\section{Disclosure}

The authors have declared that no competing interests exists.

\section{References}

1. Ayinde KS, Olaoba OT, Ibrahim B, et al. AMPK allostery: a therapeutic target for the management/treatment of diabetic nephropathy. Life Sci. 2020;261:118455. doi:10.1016/j. 1fs. 2020.118455

2. Liu M, Liang K, Zhen J, et al. Sirt6 deficiency exacerbates podocyte injury and proteinuria through targeting Notch signaling. Nat Commun. 2017;8(1):413. doi:10.1038/s41467-017-00498-4

3. Dai H, Liu Q, Liu B. Research progress on mechanism of podocyte depletion in diabetic nephropathy. $J$ Diabetes Res. 2017;2017:2615286. doi:10.1155/2017/2615286

4. Nishad R, Mukhi D, Tahaseen SV, Mungamuri SK, Pasupulati AK. Growth hormone induces Notch1 signaling in podocytes and contributes to proteinuria in diabetic nephropathy. J Biol Chem. 2019;294 (44):16109-16122. doi:10.1074/jbc.RA119.008966

5. Man SM, Karki R, Kanneganti TD. Molecular mechanisms and functions of pyroptosis, inflammatory caspases and inflammasomes in infectious diseases. Immunol Rev. 2017;277(1):61-75. doi:10.1111/ imr. 12534

6. Jorgensen I, Lopez JP, Laufer SA, Miao EA. IL-1beta, IL-18, and eicosanoids promote neutrophil recruitment to pore-induced intracellular traps following pyroptosis. Eur J Immunol. 2016;46 (12):2761-2766. doi:10.1002/eji.201646647

7. Dong T, Liao D, Liu X, Lei X. Using small molecules to dissect non-apoptotic programmed cell death: necroptosis, ferroptosis, and pyroptosis. Chembiochem. 2015;16(18):2557-2561. doi:10.1002/ cbic. 201500422

8. Xia X, Wang X, Zheng Y, Jiang J, Hu J. What role does pyroptosis play in microbial infection? J Cell Physiol. 2018;234:7885-7892. doi:10.1002/jcp.27909

9. Zeng C, Wang R, Tan H. Role of pyroptosis in cardiovascular diseases and its therapeutic implications. Int J Biol Sci. 2019;15 (7):1345-1357. doi:10.7150/ijbs.33568

10. Ruan J, Wang S, Wang J. Mechanism and regulation of pyroptosis-mediated in cancer cell death. Chem Biol Interact. 2020;323:109052. doi:10.1016/j.cbi.2020.109052

11. Wadie W, El-Tanbouly DM. Vinpocetine mitigates proteinuria and podocytes injury in a rat model of diabetic nephropathy. Eur J Pharmacol. 2017;814:187-195. doi:10.1016/j.ejphar.2017.08.027

12. Uruno A, Yagishita Y, Yamamoto M. The Keap1-Nrf2 system and diabetes mellitus. Arch Biochem Biophys. 2015;566:76-84. doi:10.1016/j.abb.2014.12.012

13. Hu Q, Zhang T, Yi L, Zhou X, Mi M. Dihydromyricetin inhibits NLRP3 inflammasome-dependent pyroptosis by activating the Nrf2 signaling pathway in vascular endothelial cells. Biofactors. 2018;44 (2):123-136. doi:10.1002/biof.1395

14. Chen X, Wei SY, Li JS, et al. Overexpression of heme oxygenase-1 prevents renal interstitial inflammation and fibrosis induced by unilateral ureter obstruction. PLoS One. 2016;11(1):e0147084. doi:10.1371/journal.pone.0147084

15. Chang TT, Chen YA, Li SY, Chen JW. Nrf-2 mediated heme oxygenase-1 activation contributes to the anti-inflammatory and renal protective effects of Ginkgo biloba extract in diabetic nephropathy. J Ethnopharmacol. 2020;266:113474. doi:10.1016/j. jep. 2020.113474 
16. Su L, Cao P, Wang H. Tetrandrine mediates renal function and redox homeostasis in a streptozotocin-induced diabetic nephropathy rat model through Nrf2/HO-1 reactivation. Ann Transl Med. 2020;8 (16):990. doi:10.21037/atm-20-5548

17. Zeng R, Zhang R, Song X, et al. The long non-coding RNA MALAT1 activates Nrf2 signaling to protect human umbilical vein endothelial cells from hydrogen peroxide. Biochem Biophys Res Commun. 2018;495(4):2532-2538. doi:10.1016/j.bbrc.2017.12.105

18. Carlomosti F, D'Agostino M, Beji S, et al. Oxidative stress-induced miR-200c disrupts the regulatory loop among SIRT1, FOXO1, and eNOS. Antioxid Redox Signal. 2017;27(6):328-344. doi:10.1089/ ars.2016.6643

19. Li Q, Zhang C, Chen R, et al. Disrupting MALAT1/miR-200c sponge decreases invasion and migration in endometrioid endometrial carcinoma. Cancer Lett. 2016;383(1):28-40. doi:10.1016/j. canlet.2016.09.019

20. Zhou S, Zhao P, Li Y, Deng T, Tian L, Li H. Renoprotective effect of atorvastatin on STZ-diabetic rats through attenuating kidney-associated dysmetabolism. Eur $J$ Pharmacol. 2014;740:9-14. doi:10.1016/j.ejphar.2014.06.055

21. Erqou S, Lee CC, Adler AI. Statins and glycaemic control in individuals with diabetes: a systematic review and meta-analysis. Diabetologia. 2014;57(12):2444-2452. doi:10.1007/s00125-014-3374-x

22. Hammad MA, Abdo MS, Mashaly AM, et al. The statins effects on HbAlc control among diabetic patients: an umbrella review of systematic reviews and meta-analyses of observational studies and clinical trials. Diabetes Metab Syndr. 2019;13(4):2557-2564. doi:10.1016/j.dsx.2019.07.005

23. Dormuth CR, Filion KB, Paterson JM, et al. Higher potency statins and the risk of new diabetes: multicentre, observational study of administrative databases. BMJ. 2014;348:g3244. doi:10.1136/bmj.g3244

24. Thongtang N, Tangkittikasem N, Samaithongcharoen K, Piyapromdee J, Srinonprasert V, Sriussadaporn S. Effect of switching from low-dose simvastatin to high-dose atorvastatin on glucose homeostasis and cognitive function in type 2 diabetes. Vasc Health Risk Manag. 2020;16:367-377. doi:10.2147/VHRM.S270751

25. Thongtang N, Ai M, Otokozawa S, et al. Effects of maximal atorvastatin and rosuvastatin treatment on markers of glucose homeostasis and inflammation. Am J Cardiol. 2011;107(3):387-392. doi:10.1016/ j.amjcard.2010.09.031

26. Henriksbo BD, Tamrakar AK, Phulka JS, Barra NG, Schertzer JD. Statins activate the NLRP3 inflammasome and impair insulin signaling via p38 and mTOR. Am J Physiol Endocrinol Metab. 2020;319 (1):E110-E116. doi:10.1152/ajpendo.00125.2020

27. Henriksbo BD, Lau TC, Cavallari JF, et al. Fluvastatin causes NLRP3 inflammasome-mediated adipose insulin resistance. Diabetes. 2014;63(11):3742-3747. doi:10.2337/db13-1398

28. Henriksbo BD, Tamrakar AK, Xu J, et al. Statins promote interleukin-1beta-dependent adipocyte insulin resistance through lower prenylation, not cholesterol. Diabetes. 2019;68(7):1441-1448. doi:10.2337/db18-0999

29. Burton DGA, Faragher RGA. Obesity and type-2 diabetes as inducers of premature cellular senescence and ageing. Biogerontology. 2018;19(6):447-459. doi:10.1007/s10522-018-9763-7

30. Guo J, Zheng HJ, Zhang W, et al. Accelerated kidney aging in diabetes mellitus. Oxid Med Cell Longev. 2020;2020:1234059. doi:10.1155/2020/1234059

31. Gupta S, Goyal P, Feinn RS, Mattana J. Role of vitamin D and its analogues in diabetic nephropathy: a meta-analysis. Am J Med Sci. 2019;357(3):223-229. doi:10.1016/j.amjms.2018.12.005

32. Esfandiari A, Pourghassem Gargari B, Noshad H, et al. The effects of vitamin D3 supplementation on some metabolic and inflammatory markers in diabetic nephropathy patients with marginal status of vitamin D: a randomized double blind placebo controlled clinical trial. Diabetes Metab Syndr. 2019;13(1):278-283. doi:10.1016/j. dsx.2018.09.013
33. Shi J, Gao W, Shao F. Pyroptosis: gasdermin-mediated programmed necrotic cell death. Trends Biochem Sci. 2017;42(4):245-254. doi:10.1016/j.tibs.2016.10.004

34. Doitsh G, Galloway NL, Geng X, et al. Corrigendum: cell death by pyroptosis drives CD4 T-cell depletion in HIV-1 infection. Nature. 2017;544(7648):124. doi:10.1038/nature22066

35. Li X, Zeng L, Cao C, et al. Long noncoding RNA MALAT1 regulates renal tubular epithelial pyroptosis by modulated miR-23c targeting of ELAVL1 in diabetic nephropathy. Exp Cell Res. 2017;350 (2):327-335. doi:10.1016/j.yexcr.2016.12.006

36. Xie C, Wu W, Tang A, Luo N, Tan Y. IncRNA GAS5/miR-452-5p reduces oxidative stress and pyroptosis of high-glucose-stimulated renal tubular cells. Diabetes Metab Syndr Obes. 2019;12:2609-2617. doi:10.2147/DMSO.S228654

37. Liu C, Zhuo H, Ye MY, Huang GX, Fan M, Huang XZ. LncRNA MALAT1 promoted high glucose-induced pyroptosis of renal tubular epithelial cell by sponging miR-30c targeting for NLRP3. Kaohsiung J Med Sci. 2020;36(9):682-691. doi:10.1002/kjm2.12226

38. An X, Zhang Y, Cao Y, Chen J, Qin H, Yang L. Punicalagin protects diabetic nephropathy by inhibiting pyroptosis based on TXNIP/ NLRP3 pathway. Nutrients. 2020;12(5):1516. doi:10.3390/ nu12051516

39. Ke R, Wang Y, Hong S, Xiao L. Endoplasmic reticulum stress related factor IRE1alpha regulates TXNIP/NLRP3-mediated pyroptosis in diabetic nephropathy. Exp Cell Res. 2020;396(2):112293. doi:10.1016/j.yexcr.2020.112293

40. Fang Y, Tian S, Pan Y, et al. Pyroptosis: a new frontier in cancer. Biomed Pharmacother. 2020;121:109595. doi:10.1016/j.biopha.20 19.109595

41. Qiu YY, Tang LQ. Roles of the NLRP3 inflammasome in the pathogenesis of diabetic nephropathy. Pharmacol Res. 2016;114:251-264. doi:10.1016/j.phrs.2016.11.004

42. Xin R, Sun X, Wang Z, et al. Apocynin inhibited NLRP3/XIAP signalling to alleviate renal fibrotic injury in rat diabetic nephropathy. Biomed Pharmacother. 2018;106:1325-1331. doi:10.1016/j.biopha.2018.07.036

43. Lu T, Sun X, Li Y, Chai Q, Wang XL, Lee HC. Role of Nrf2 signaling in the regulation of vascular BK channel betal subunit expression and BK channel function in high-fat diet-induced diabetic mice. Diabetes. 2017;66(10):2681-2690. doi:10.2337/db17-0181

44. Jimenez-Osorio AS, Gonzalez-Reyes S, Garcia-Nino WR, et al. Association of nuclear factor-erythroid 2-related factor 2, thioredoxin interacting protein, and heme oxygenase-1 gene polymorphisms with diabetes and obesity in Mexican patients. Oxid Med Cell Longev. 2016;2016:7367641. doi:10.1155/2016/7367641

45. Mathur A, Pandey VK, Kakkar P. Activation of GSK3beta/ beta-TrCP axis via PHLPP1 exacerbates Nrf2 degradation leading to impairment in cell survival pathway during diabetic nephropathy. Free Radic Biol Med. 2018;120:414-424. doi:10.1016/j. freeradbiomed.2018.04.550

46. Diao C, Chen Z, Qiu T, et al. Inhibition of PRMT5 attenuates oxidative stress-induced pyroptosis via activation of the Nrf2/HO-1 signal pathway in a mouse model of renal ischemia-reperfusion injury. Oxid Med Cell Longev. 2019;2019:2345658. doi:10.1155/ 2019/2345658

47. Mu Z, Zhang H, Lei P. Piceatannol inhibits pyroptosis and suppresses oxLDL-induced lipid storage in macrophages by regulating miR-200a/Nrf2/GSDMD axis. Biosci Rep. 2020;40(9). doi:10.1042/ BSR20201366

48. Weidle UH, Birzele F, Kollmorgen G, Ruger R. Long non-coding RNAs and their role in metastasis. Cancer Genomics Proteomics. 2017;14(3):143-160. doi:10.21873/cgp.20027

49. Ou C, Sun Z, He X, et al. Targeting YAP1/LINC00152/FSCN1 signaling axis prevents the progression of colorectal cancer. Adv Sci (Weinh). 2020;7(3):1901380. doi:10.1002/advs.201901380 
50. Zhang X, Shang J, Wang X, et al. Microarray analysis reveals long noncoding RNA SOX2OT as a novel candidate regulator in diabetic nephropathy. Mol Med Rep. 2018;18(6):5058-5068. doi:10.3892/ mmr.2018.9534

51. Shang J, Wang S, Jiang Y, et al. Identification of key lncRNAs contributing to diabetic nephropathy by gene co-expression network analysis. Sci Rep. 2019;9(1):3328. doi:10.1038/s41598-019-39298-9

52. Gao J, Wang W, Wang F, Guo C. LncRNA-NR_033515 promotes proliferation, fibrogenesis and epithelial-to-mesenchymal transition by targeting miR-743b-5p in diabetic nephropathy. Biomed Pharmacother. 2018;106:543-552. doi:10.1016/j.biopha.2018.06.104

53. Zhang X, Hamblin MH, Yin KJ. The long noncoding RNA Malat1: its physiological and pathophysiological functions. RNA Biol. 2017;14(12):1705-1714. doi:10.1080/15476286.2017.1358347
54. Su Y, Wu H, Pavlosky A, et al. Regulatory non-coding RNA: new instruments in the orchestration of cell death. Cell Death Dis. 2016;7 (8):e2333. doi:10.1038/cddis.2016.210

55. Ma X, Cen S, Wang L, et al. Genome-wide identification and comparison of differentially expressed profiles of miRNAs and lncRNAs with associated ceRNA networks in the gonads of Chinese soft-shelled turtle, Pelodiscus sinensis. BMC Genomics. 2020;21 (1):443. doi:10.1186/s12864-020-06826-1

56. Cortez MA, Valdecanas D, Zhang X, et al. Therapeutic delivery of miR-200c enhances radiosensitivity in lung cancer. Mol Ther. 2014;22(8):1494-1503. doi:10.1038/mt.2014.79

Diabetes, Metabolic Syndrome and Obesity: Targets and Therapy

\section{Publish your work in this journal}

Diabetes, Metabolic Syndrome and Obesity: Targets and Therapy is an international, peer-reviewed open-access journal committed to the rapid publication of the latest laboratory and clinical findings in the fields of diabetes, metabolic syndrome and obesity research. Original research, review, case reports, hypothesis formation, expert opinion and commentaries are all considered for publication. The manuscript management system is completely online and includes a very quick and fair peer-review system, which is all easy to use. Visit http://www.dovepress.com/testimonials.php to read real quotes from published authors.

Submit your manuscript here: https://www.dovepress.com/diabetes-metabolic-syndrome-and-obesity-targets-and-therapy-journal 